\begin{tabular}{|r|l|}
\hline \multicolumn{2}{|c|}{ Statistica Sinica Preprint No: SS-2021-0036 } \\
\hline Title & $\begin{array}{l}\text { Regression Analysis of Panel Count Data With Both } \\
\text { Time-Dependent Covariates and Time-Varying Effects }\end{array}$ \\
\hline Manuscript ID & SS-2021-0036 \\
\hline URL & http://www.stat.sinica.edu.tw/statistica/ \\
\hline DOI & $10.5705 /$ ss.202021.0036 \\
\hline Complete List of Authors & $\begin{array}{l}\text { Yuanyuan Guo, } \\
\text { Dayu Sun and } \\
\text { Jianguo Sun }\end{array}$ \\
\hline Corresponding Author & Jianguo Sun \\
\hline E-mail & sunj@missouri.edu \\
\hline Notice: Accepted version subject to English editing.
\end{tabular}




\title{
REGRESSION ANALYSIS OF PANEL COUNT DATA WITH BOTH TIME-DEPENDENT COVARIATES
} AND TIME-VARYING EFFECTS

\author{
Yuanyuan Guo ${ }^{1 *}$, Dayu Sun ${ }^{2 *}$ and Jianguo Sun ${ }^{1}$ \\ ${ }^{1}$ University of Missouri and ${ }^{2}$ Emory University
}

\begin{abstract}
Panel count data occur in many fields including clinical, demographic and industrial studies, and extensive literature has been established for their regression analysis. However, most of the existing methods apply only to the situations where both covariates and their effects are constant or one of them may be time-dependent. This paper considers a situation where both covariates and their effects may be time-dependent, and an estimating equationbased approach is developed for estimating these time-varying effects. In the method, the B-splines are employed to approximate time-dependent coefficients, and the asymptotic properties of the proposed estimators are established. To assess the finite sample performance of the proposed estimators, an extensive simulation study is conducted and suggests that the proposed method works well in practical situations. An application to the China Health and Nutrition Survey is provided.
\end{abstract}

Keywords and phrases: B-spline, Panel count data, Proportional mean model, Time-dependent effect.

*Equal contribution. 


\section{Introduction}

Event history studies that concern the times to recurrent event occurrences often appear in many fields, including clinical, demographic and industrial studies. In these situations, two types of data commonly arise and they are recurrent event data and panel count data (Cook and Lawless, 2007; Sun and Zhao, 2013). The former means that all study subjects can be observed or followed continuously and thus one has complete data on the occurrence times of the event of interest. In contrast, the latter means that study subjects can be observed only at discrete time points and only incomplete information is available on the occurrence times. Despite the missing information, panel count data frequently arise in practice as the subjects usually cannot be followed continuously in reality. Thus it is necessary to develop statistical methods for panel count data due to its prevalence.

An example of panel count data that motivated this study is given by the China Health and Nutrition Survey (CHNS) (Tian, 2018; Oliveira, 2016). The surveys were conducted every 2 to 4 years between 1989 and 2015 to obtain the fertility histories of the female participants and their demographic, education, income, and health information. Due to the intermittent survey times, the exact dates of the pregnancies or childbirths may not be available, leading to typical panel count data on the pregnancy number of the study subjects. Among others, one objective of the CHNS is to determine the relationship between the pregnancy process and various 
factors or covariates, including the income, location, education level and health status of the female subject. It is apparent that some of these factors may change over time, and furthermore, their effects on the pregnancy process may change over time too. For instance, the fertility difference between well-educated and ill-educated women may be more considerable for older women as the education effect could accumulate along with the lives of the female. Additionally, the health condition could also have a larger impact on older women as they are less resilient to diseases. As pointed out by many authors (Tian et al., 2005; Yu and Lin, 2010; Perperoglou, 2013; Lin et al., 2015), the analysis of such data that ignores the time-dependency nature of covariates and/or their effects would be less efficient at least. In other words, one needs a statistical method that can accommodate both time-dependent covariates and time-varying effects for the analysis.

A large literature has been established for regression analysis of panel count data with time-independent covariates and covariate effects. Also some methods have been proposed for the situations where either covariates are time-dependent or their effects are time-varying. For example, Li et al. (2010) considered a semiparametric transformation mean model for the panel count data with time-dependent covariates. Zhao et al. (2018) and Wang and Yu (2021) considered the situation of time-varying coefficients and proposed some pseudo-likelihood methods for estimation. The former used the B-spline function approximation and the latter employed 
the local polynomial approximation. However, both methods can only apply to constant covariate situations. To the best of our knowledge, it does not seem to exist an established method for regression analysis of panel count data that allows both time-dependent covariates and time-varying effects. Corresponding to this, in the following, an estimating equation-based method will be proposed.

The remainder of this paper is organized as follows. After introducing the notation and assumptions that will be used throughout the paper, an estimating equation procedure will be presented in Section 2 for the estimation of covariate effects. In the proposed method, the conditional mean model is employed for the underlying recurrent event process and B-spline functions are used to approximate time-varying covariate effects. The asymptotic properties of the proposed estimators, namely, the consistency, convergence rate and asymptotic distribution, will be established in Section 3. Section 4 will present some results obtained from an extensive simulation study conducted to assess the finite sample performance of the proposed method, and they suggest that it works well for practical situations. In Section 5, we apply the proposed approach to the CHNS described above, and Section 6 gives some discussion and concluding remarks. 


\section{Estimation of Time-varying Covariate Effects}

Consider a recurrent event study consisting of $n$ independent subjects and let $N_{i}(t)$ denote the underlying recurrent event process representing the total number of the occurrences of the recurrent event of interest up to time $t$ for subject $i$. Assume that $N_{i}(t)$ is potentially observed only at $0<t_{i, 1}<\cdots<t_{i, m_{i}}<\tau$ and define $H_{i}^{*}(t)=$

$\sum_{j=1}^{m_{i}} I\left(t_{i, j} \leq t\right)$, the underlying observation process. In practice, for each subject, there usually exists a following or stopping time $C_{i}$, and it follows that the realized censored observation process on the $i$ th subject is given by $H_{i}(t)=H_{i}^{*}\left\{\min \left(C_{i}, t\right)\right\}$. In other words, one only observes panel count data such that $N_{i}(t)$ is observed only at the time points where $H_{i}(t)$ jumps, $i=1, \ldots, n$.

For each subject, suppose that there exist two vectors of covariates denoted by $\mathbf{W}_{i}(t)=\left(W_{i 1}(t), \ldots, W_{i p_{1}}(t)\right)^{T}$ and $\mathbf{Z}_{i}(t)=\left(Z_{i 1}(t), \ldots, Z_{i p_{2}}(t)\right)^{T}$ that are possibly time-dependent. The former $\mathbf{W}_{i}(t)$ represents the covariates assumed to have constant effects, while the latter $\mathbf{Z}_{i}(t)$ denotes the covariates assumed to have timevarying effects. To describe the covariate effects on $N_{i}(t)$, we assume that given $\mathbf{W}_{i}(t)$ and $\mathbf{Z}_{i}(t)$, the recurrent event process $N_{i}(t)$ follows the conditional multiplicative mean model

$$
E\left\{N_{i}(t) \mid \mathbf{W}_{i}(t), \mathbf{Z}_{i}(t)\right\}=\Lambda(t) \exp \left\{\boldsymbol{\gamma}^{T} \mathbf{W}_{i}(t)+\boldsymbol{\beta}^{T}(t) \mathbf{Z}_{i}(t)\right\}
$$


In the above, $\Lambda(t)$ denotes an unknown baseline mean function, and $\gamma=\left(\gamma_{1}, \ldots, \gamma_{p_{1}}\right)^{T}$ and $\boldsymbol{\beta}(t)=\left(\beta_{1}(t), \ldots, \beta_{p_{2}}(t)\right)^{T}$ represent constant and time-dependent coefficients, respectively. Besides, we will assume that given $\mathbf{W}_{i}(t)$ and $\mathbf{Z}_{i}(t), N_{i}(t)$ and $H_{i}(t)$ are conditionally independent and some comments on this will be given in Section 6 .

Let $\mathcal{M}_{j}$ denote the parameter space for $\beta_{j}, j=1, \ldots, p_{2}$. Due to the infinite dimension of each $\mathcal{M}_{j}$, the estimation of $\boldsymbol{\beta}(t)$ is usually infeasible. To deal with this, we propose to employ the sieve approach to approximate $\boldsymbol{\beta}(t)$ by a linear combination of a finite number of basis functions such as B-splines (He et al., 2017). More specifically, define a sequence of knots $\mathcal{T}=\left\{t_{j}\right\}_{j=1}^{m_{n}+2 l}$ with $0=t_{1}=\cdots=t_{l}<t_{l+1}<$ $\cdots<t_{m_{n}+l}<t_{m_{n}+l+1}=\cdots=t_{m_{n}+2 l}=\tau$ that partition $[0, \tau]$ into $K_{n}+1$ subintervals $\left[t_{l+j}, t_{l+j+1}\right]$ for $j=0, \ldots, K_{n}$, where $K_{n}=O\left(n^{\nu}\right)$ and $\max _{0<j<m_{n}}\left|t_{j+1}-t_{j}\right|=$ $O\left(n^{-\nu}\right)$ for $\nu \in(0,0.5)$. For simplicity, we assume the same knots for all $\beta_{j}(t)$ for $j=1, \ldots, p_{2}$. Then we can construct a sieve space to approximate $\mathcal{M}_{j}$ as

$$
\mathcal{M}_{n j}=\left\{\beta_{n j}(t)=\alpha_{j 0}+\sum_{k=1}^{q_{n}} \alpha_{j k} B_{k}(t)=\mathbf{B}_{n}^{T}(t) \boldsymbol{\alpha}_{j},\left\|\boldsymbol{\alpha}_{j}\right\|_{\infty}<M_{n}\right\}
$$

the class of B-splines of order $l$ with the knots sequence $\mathcal{T}$. In the above, $M_{n}$ is some large number with $M_{n} \rightarrow \infty$ as $n \rightarrow \infty, q_{n}=K_{n}+l, \mathbf{B}_{n}(t)=\left\{1, B_{1}(t), \ldots, B_{k}(t)\right\}^{T}$ is a class of B-spline basis, and $\boldsymbol{\alpha}_{n j}=\left(\alpha_{n j 0}, \alpha_{n j 1}, \ldots \alpha_{n j q_{n}}\right)$. Since the dimension of $\mathcal{M}_{n j}, q_{n}$, is finite, we can now estimate the parameters in a much easier way by 
adopting the existing methods for panel count data with a finite number of parameters.

Under the sieve space, by replacing $\boldsymbol{\beta}(t)$ by $\boldsymbol{\beta}_{n}(t)$, model (2.1) can be rewritten as

$$
\begin{array}{r}
E\left\{N_{i}(t) \mid \mathbf{W}_{i}(t), \mathbf{Z}_{i}(t)\right\}=\Lambda_{0}(t) \exp \left\{\boldsymbol{\gamma}^{T} \mathbf{W}_{i}(t)+\sum_{j=1}^{p_{2}}\left(\mathbf{B}_{n}^{T}(t) \boldsymbol{\alpha}_{n j}\right) Z_{i j}(t)\right\} \\
=\Lambda_{0}(t) \exp \left\{\boldsymbol{\gamma}^{T} \mathbf{W}_{i}(t)+\boldsymbol{\alpha}_{n}^{T} \tilde{\mathbf{Z}}_{i}(t)\right\}=\Lambda_{0}(t) \exp \left\{\boldsymbol{\theta}_{n}^{T} \mathbf{X}_{i}(t)\right\}
\end{array}
$$

Here $\tilde{\mathbf{Z}}_{i}(t)=\left(Z_{i 1}(t) \mathbf{B}_{n}^{T}(t), Z_{i 2}(t) \mathbf{B}_{n}^{T}(t), \ldots, Z_{i p_{2}}(t) \mathbf{B}_{n}^{T}(t)\right)^{T}, \boldsymbol{\alpha}_{n}=\left(\boldsymbol{\alpha}_{n 1}^{T}, \boldsymbol{\alpha}_{n 1}^{T}, \ldots, \boldsymbol{\alpha}_{n p_{2}}^{T}\right)^{T}$ $\mathbf{X}_{i}(t)=\left(\mathbf{W}_{i}^{T}(t), \tilde{\mathbf{Z}}_{i}^{T}(t)\right)^{T}$, and $\boldsymbol{\theta}_{n}=\left(\boldsymbol{\gamma}, \boldsymbol{\alpha}_{n}\right)^{T}$. Note that model (2.2) involves only time-independent covariate effects and many existing methods could be adopted to estimate $\boldsymbol{\theta}_{n}$ without much modification. More specifically, motivated by Hu et al. (2003), we propose to use the estimating equation

$$
\frac{1}{n} \sum_{i=1}^{n} \int_{0}^{\tau} Y_{i}(t) N_{i}(t)\left\{\mathbf{X}_{i}(t)-\overline{\mathbf{X}}\left(t ; \boldsymbol{\theta}_{n}\right)\right\} d H_{i}(t)=0
$$

In the above, $Y_{i}(t)=I\left(C_{i} \geq t\right)$ is the at-risk indicator and $\overline{\mathbf{X}}\left(t ; \boldsymbol{\theta}_{n}\right)=\mathbf{S}_{1}\left(t ; \boldsymbol{\theta}_{n}\right) / S_{0}\left(t ; \boldsymbol{\theta}_{n}\right)$, where

$$
S_{u}(t ; \boldsymbol{\theta})=\frac{1}{n} \sum_{i=1}^{n} Y_{i}(t) \mathbf{X}_{i}^{\otimes u}(t) \exp \left(\boldsymbol{\theta}^{T} X_{i}(t)\right) d H_{i}(t)
$$

$u=0,1,2$ for $0 \leq t \leq \tau$, with $a^{\otimes 0}=1, a^{\otimes 1}=a$ and $a^{\otimes 2}=a a^{T}$ for some vector $a$. 
Let $\hat{\boldsymbol{\theta}}_{n}$ denote the estimator of $\boldsymbol{\theta}_{n}$ given by the solution to the equation (2.3). Then one can estimate $\beta_{j}(t)$ by $\hat{\beta}_{j}(t)=\mathbf{B}_{n}^{T}(t) \hat{\boldsymbol{\alpha}}_{n j}$. In practice, sometimes one may also be interested in estimating the baseline mean function $\Lambda(t)$ and for this, it is apparent that one natural estimator is given by the Breslow-type estimator

$$
\hat{\Lambda}\left(t, \hat{\boldsymbol{\theta}}_{n}\right)=\sum_{i=1}^{n} \frac{Y_{i}(u) N_{i}(u) d H_{i}(u)}{n S_{0}\left(u ; \hat{\boldsymbol{\theta}}_{n}\right)} .
$$

\section{Asymptotic Properties}

Now we will establish the asymptotic properties of the estimators proposed in the previous section, including the consistency, convergence rate and asymptotic normality. Let $\boldsymbol{\vartheta}=(\boldsymbol{\gamma}, \boldsymbol{\beta}, \Lambda), \hat{\boldsymbol{\vartheta}}_{n}=\left(\hat{\boldsymbol{\gamma}}, \hat{\boldsymbol{\beta}}_{n}, \hat{\boldsymbol{\Lambda}}\right)$, and $\boldsymbol{\vartheta}_{0}=\left(\boldsymbol{\gamma}_{0}, \boldsymbol{\beta}_{0}, \Lambda_{0}\right)$ denote the true value of $\boldsymbol{\vartheta}$. Also for convenience, let $\mathbf{V}(t)=\left(\mathbf{W}^{T}(t), \mathbf{Z}^{T}(t)\right)^{T}$, the population version of the combined covariates, and define the parameter space $\Theta=\mathcal{A} \times \mathcal{M} \times \mathcal{F}$, where $\mathcal{A}, \mathcal{M}=\prod_{j=1}^{p_{2}} \mathcal{M}_{j}$ and $\mathcal{F}$ denote the parameter spaces of $\gamma, \boldsymbol{\beta}$ and $\Lambda$, respectively. Let $\mathfrak{B}^{d}$ denote the collection of Borel sets in $\mathbb{R}^{d}$ and $\mathfrak{L}_{2}[0, \tau]$ the collection of Borel sets in $\mathcal{L}_{2}$ on $[0, \tau]$, and define $\mathfrak{B}^{1}[0, \tau]=\left\{B \cap[0, \tau]: B \in \mathfrak{B}^{1}\right\}$ and $\mathfrak{L}_{2}^{d}[0, \tau]=\underbrace{\mathfrak{L}_{2}[0, \tau] \times \ldots \times \mathfrak{L}_{2}[0, \tau]}_{d}$

Also define the measure

$$
v_{1}\left(B_{1} \times B_{2} \times B_{3}\right)=\int_{B_{3} \times B_{2}} \int_{B_{1}} d E[Y(t) H(t)] d \mu_{Z \times W}
$$


for $B_{1} \in \mathfrak{B}^{1}[0, \tau], B_{2} \in \mathfrak{L}_{2}^{p_{2}}[0, \tau]$ and $B_{3} \in \mathfrak{L}_{2}^{p_{1}}[0, \tau]$, where $\mu_{Z \times W}$ denotes the joint probability measure for $\mathbf{W}(t)$ and $\mathbf{Z}(t)$. Alternatively, by the definition of $\mathbf{V}$, we have $\mu_{V}=\mu_{Z \times W}$ and can rewrite $v_{1}\left(B_{1} \times B_{2} \times B_{3}\right)$ as

$$
v_{1}\left(B_{1} \times B_{4}\right)=\int_{B_{4}} \int_{B_{1}} d E[Y(t) H(t)] d \mu_{V}
$$

for $B_{1} \in \mathfrak{B}^{1}[0, \tau]$ and $B_{4} \in \mathfrak{L}_{2}^{p}[0, \tau]$; and $\mu_{1}(B)=v_{1}\left(B \times \mathfrak{L}_{2}^{p}[0, \tau]\right)$. Define the $L_{2}$ metric $d\left(\boldsymbol{\vartheta}_{1}, \boldsymbol{\vartheta}_{2}\right)$ on $\Theta$ as

$$
\begin{aligned}
d\left(\boldsymbol{\vartheta}_{1}, \boldsymbol{\vartheta}_{2}\right)^{2}=\left\|\boldsymbol{\gamma}_{1}-\boldsymbol{\gamma}_{2}\right\|_{2}^{2}+\int\left\|\boldsymbol{\beta}_{1}(u)-\boldsymbol{\beta}_{2}(u)\right\|_{2}^{2} d \mu_{1}(u) & \\
& +\int\left(\Lambda_{1}(u)-\Lambda_{2}(u)\right)^{2} d \mu_{1}(u) .
\end{aligned}
$$

To establish the asymptotic results, we need the following regularity conditions.

(C1) The observation process has the rate function $E\left[d H^{*}(t) \mid \mathbf{W}(t), \mathbf{Z}(t), C\right]=$ $\omega(t) d t$, where $\omega(t)$ is a bounded, nonnegative and continuous function on $[0, \tau]$. There exists a positive integer $D_{0}$ such that $\operatorname{Pr}\left(H^{*}(\tau)<D_{0}\right)=1$. That is, the total observation number is finite. Moreover, the support of $\omega(t)$ is $\left[\tau_{0}, \tau\right]$ with $\tau_{0}>0$ and $\Lambda_{0}\left(\tau_{0}\right)>0$ for some constant $\tau_{0}$.

(C2) The measure $\mu_{1} \times \mu_{V}$ is absolutely continuous with respect to $v_{1}$ and $\mu_{1}(\{\tau\})>$ 0. 
(C3) The parameters space of $\Lambda, \mathcal{F}$, consists of bounded non-decreasing functions in $\mathcal{L}_{2}$ over $[0, \tau]$.

(C4) The parameters space of $\boldsymbol{\beta}_{j}, \mathcal{M}_{j}$, is bounded and convex in $\mathcal{L}_{2}([0, \tau])$ for each $j=1, \ldots, p_{2}$. Each component of the true value of $\boldsymbol{\beta}(t)$, denoted by $\beta_{0 j}(t)$, $j=1, \ldots, p_{2}$, is continuously $r$ th differentiable in $[0, \tau]$ for $r+1 \leq l$.

(C5) The parameter space of $\boldsymbol{\gamma}, \mathcal{A}$, is bounded and convex in $\mathbb{R}^{p_{1}}$.

(C6) The covariate vector $\mathbf{V}(t)=\left(\mathbf{W}^{T}(t), \mathbf{Z}^{T}(t)\right)^{T}$ is uniformly bounded over $[0, \tau]$ with the distribution $\mu_{V}$.

(C7) Given $\mathbf{V}(t), t \in[0, \tau], C$ and $N$ are independent. Besides, with probability 1 ,

$$
\begin{aligned}
\inf _{\mathbf{V}(t), t \in[0, \tau]} \operatorname{Pr}(C \geq \tau \mid \mathbf{V}(t) & =\mathbf{v}(t), t \in[0, \tau]) \\
& =\inf _{\mathbf{V}(t), t \in[0, \tau]} \operatorname{Pr}(C=\tau \mid \mathbf{V}(t)=\mathbf{v}(t), t \in[0, \tau])>0 .
\end{aligned}
$$

(C8) If $\boldsymbol{\gamma}^{T} \mathbf{W}(t)+\boldsymbol{\beta}^{T}(t) \mathbf{Z}(t) \equiv 0$ for $t \in[0, \tau]$ with probability 1 for some $\boldsymbol{\gamma}$ and $\boldsymbol{\beta}$, then $\boldsymbol{\gamma}=0$ and $\boldsymbol{\beta}(t)=0$ for $t \in[0, \tau]$ with probability 1 .

(C9) The random function $M_{0}(\mathbf{V})=\int N(t) \log (N(t)) d H(t)$ satisfies $E\left[M_{0}(\mathbf{V})\right]<$ $\infty$

(C10) $E[\exp (c N(t))]$ is bounded in $[0, \tau]$ for some constant $c>0$. 
(C11) The true baseline mean function $\Lambda_{0}$ is differentiable in $\left[\tau_{0}, \tau\right]$. Moreover, the lower and upper bounds of its first order derivative are positive and finite on $\left[\tau_{0}, \tau\right]$

(C12) There exist $\eta_{1} \in(0,1)$ such that $a^{T} \operatorname{Var}(\mathbf{V}(U) \mid U) a \geq \eta_{1} a^{T} E\left(\mathbf{V}(U) \mathbf{V}^{T}(U) \mid U\right) a$, a.s. for all $a \in \mathbb{R}^{p_{1}+p_{2}}$, where $(U, \mathbf{V})$ has distribution $\nu_{1} / \nu_{1}\left(\mathbb{R}^{+} \times \mathcal{V}\right)$ where $\mathcal{V}$ is the support of $\mathbf{V}$.

Note that conditions $(\mathrm{C} 1)$ and $(\mathrm{C} 7)$ are common on the observation schemes and similar to the combination of C8, C10 and C11 in Lu et al. (2009). Condition (C2) comes from the condition in Theorem 1 of Wellner and Zhang (2007) and Theorem 1 of Lu et al. (2009), ensuring that $\hat{\Lambda}$ is bounded, and conditions (C6)-(C11) are common assumptions in the semiparametric estimation. Also conditions (C2) and (C8) ensure the identifiability of the semiparametric model and conditions (C9), (C10) and (C11) are technical assumptions, similar to conditions C4, C10 and C12 in Wellner and Zhang (2007). Condition (C12) is needed to prove the convergence rate and can be justified by arguments similar to those in Wellner and Zhang (2007). Now we are ready to establish the asymptotic properties of the proposed estimator.

Theorem 1 (Consistency). Assume that the regularity conditions (C1)-(C9) given above hold. Then we have that $d\left(\hat{\boldsymbol{\vartheta}}_{n}, \boldsymbol{\vartheta}_{0}\right) \rightarrow 0$ in probability as $n \rightarrow \infty$.

Note that the proof of the consistency does not rely on the continuity of the 
baseline function $\Lambda$ but requires the differentiability of the time-varying coefficients. This is because the B-spline approximation usually works well when the true timevarying coefficient functions are smooth to some degree. To derive the convergence rate in the next theorem, we need condiition (C11) to control the smoothness of $\Lambda$ as well as condition (C12).

Theorem 2 (Rate of Convergence). Assume that the regularity conditions (C1)(C12) given above hold. Then we have that

$$
n^{\min \left\{n^{\frac{1-\nu}{3}}, n^{r \nu}\right\}} d\left(\hat{\boldsymbol{\vartheta}}_{n}, \boldsymbol{\vartheta}_{0}\right)=O_{p}(1)
$$

with the optimal rate $O_{p}\left(n^{-r /(3 r+1)}\right)$ achieved at $\nu=1 /(1+3 r)$.

Note that the order of the optimal rate $n^{-r /(3 r+1)}$ is slower than $n^{-r /(2 r+1)}$ in Lu et al. (2009) because the nonparametric parameter $\Lambda$ is essentially estimated by a step function though $\boldsymbol{\beta}(t)$ is estimated by B-splines. Nevertheless, we can still derive the asymptotic distribution of $\hat{\gamma}$ with the rate of convergence $n^{-1 / 2}$. The next theorem establishes the asymptotic normality of $\hat{\boldsymbol{\theta}}_{n}$ in the form similar to He et al. (2017).

Theorem 3 (Asymptotic Normality). Assume that the regularity conditions (C1)(C12) given above hold and also $(4 r)^{-1}<\nu<2^{-1}$ with $r>1$. Define $\mathcal{H}_{1}=\left\{\boldsymbol{h}_{1}\right.$ : $\left.\boldsymbol{h}_{1} \in \mathcal{A},\left\|\boldsymbol{h}_{1}\right\| \leq 1\right\}, \mathcal{H}_{2}=\left\{\boldsymbol{h}_{2}: \boldsymbol{h}_{2} \in \mathcal{M}\right.$, each component of $\boldsymbol{h}_{2}$ is of bounded total variation. $\}$, 
and $\mathcal{H}_{3}=\left\{h_{3}: h_{3}\right.$ is a fucntion with bouned total variation in $[0, \tau]$ and $\left.h_{3}(0)=0\right\}$.

Then for some $\left(\boldsymbol{h}_{1}, \boldsymbol{h}_{2}, h_{3}\right) \in \mathcal{H}_{1} \times \mathcal{H}_{2} \times \mathcal{H}_{3}$, we have that

$\sqrt{n}\left(\hat{\boldsymbol{\gamma}}-\gamma_{0}\right)^{T} \boldsymbol{h}_{1}+\sqrt{n} \int_{0}^{\tau}\left(\hat{\boldsymbol{\beta}}(t)-\boldsymbol{\beta}_{0}(t)\right)^{T} d \boldsymbol{h}_{2}(t)+\sqrt{n} \int_{0}^{\tau}\left(\hat{\Lambda}(t)-\Lambda_{0}(t)\right) d h_{3}(t) \rightarrow_{d} N\left(0, \sigma^{2}\right)$

where $\sigma^{2}$ is given in the Supplementary Material.

The proof of the results above is sketched in the Supplementary Material and the result above suggests that one can asymptotically approximate the distribution of $\hat{\gamma}$ by the normal distribution. Note that similar to He et al. (2017), however, we cannot determine the explicit form of the asymptotic distribution because the explicit forms of $\left(\boldsymbol{h}_{1}, \boldsymbol{h}_{2}, h_{3}\right)$ cannot be determined though existing. To estimate the asymptotic covariance matrix of $\hat{\boldsymbol{\gamma}}$ and the pointwise asymptotic variance of $\hat{\boldsymbol{\beta}}(t)$ in $t \in[0, \tau]$ or the covariance matrix of $\hat{\boldsymbol{\theta}}_{n}$, based on the estimating equation (2.3) and by following Amorim et al. (2008) and Hu et al. (2003), we propose to employ the robust sandwich-type estimator $\hat{\Sigma}=\hat{\mathbf{A}}^{-1} \hat{\mathbf{D}} \hat{\mathbf{A}}^{-1}$. Here

$$
\hat{\mathbf{A}}=\left.\frac{\partial \mathbf{U}\left(\boldsymbol{\theta}_{n}\right)}{\partial \boldsymbol{\theta}_{n}}\right|_{\boldsymbol{\theta}_{\boldsymbol{n}}=\hat{\boldsymbol{\theta}}_{\boldsymbol{n}}}=-\sum_{i=1}^{n} \int_{0}^{\tau} Y_{i}(t) N_{i}(t)\left\{\frac{\mathbf{S}_{2}\left(t ; \hat{\boldsymbol{\theta}}_{\boldsymbol{n}}\right)}{S_{0}\left(t ; \hat{\boldsymbol{\theta}}_{\boldsymbol{n}}\right)}-\left(\frac{\mathbf{S}_{1}\left(t ; \hat{\boldsymbol{\theta}}_{\boldsymbol{n}}\right)}{S_{0}\left(t ; \hat{\boldsymbol{\theta}}_{\boldsymbol{n}}\right)}\right)^{\otimes 2}\right\} d H_{i}(t)
$$


and

$$
\hat{\mathbf{D}}=\sum_{i=1}^{n}\left[\int_{0}^{\tau} Y_{i}(t)\left(N_{i}(t)-\hat{\Lambda}\left(t, \hat{\boldsymbol{\theta}}_{n}\right) \exp \left(\hat{\boldsymbol{\theta}}_{\boldsymbol{n}}^{T} \mathbf{X}_{i}(t)\right)\right)\left\{\mathbf{X}(t)-\overline{\mathbf{X}}\left(t ; \hat{\boldsymbol{\theta}}_{\boldsymbol{n}}\right)\right\} d H_{i}(t)\right]^{\otimes 2}
$$

It follows from the above that the asymptotic covariance matrix of $\hat{\gamma}$ can be estimated by the top-left $p_{1} \times p_{1}$ sub-matrix of $\hat{\Sigma}$, denoted by $\hat{\Sigma}_{\hat{\gamma}}$. Furthermore, since $\hat{\beta}_{j}=\mathbf{B}_{n}^{T}(t) \hat{\boldsymbol{\alpha}}_{j}$, the pointwise asymptotic variance of $\hat{\boldsymbol{\beta}}_{j}(t)$ for a given $t$ can be estimated by $\hat{\Omega}_{j}(t)=\mathbf{B}_{n}^{T}(t) \hat{\Sigma}_{\hat{\boldsymbol{\alpha}}_{j}} \mathbf{B}_{n}(t)$, where $\hat{\Sigma}_{\hat{\boldsymbol{\alpha}}_{j}}$ denotes the diagonal block submatrix of $\hat{\Sigma}$ formed from $\left(p_{1}+(j-1) p_{2}+1\right)$ th to $\left(p_{1}+j p_{2}\right)$ th rows and columns, corresponding to the estimated covariance matrix of $\hat{\boldsymbol{\alpha}}_{j}$. The numerical study in the next section suggests that the estimators above work well. As an alternative, of course, one may estimate the asymptotic covariance matrix of $\hat{\gamma}$ by using the bootstrap procedure as shown in the next section although it is relatively more timeconsuming.

\section{A Simulation Study}

In this section, we present some results obtained from an extensive simulation study conducted to evaluate the finite sample performance of the estimation procedure proposed in the previous sections. In the study, we considered the situation of four covariates among which two have constant effects and the other two have time-varying 
effects. That is, $p_{1}=p_{2}=2$. More specifically, we assumed that $W_{1}(t)$ and $Z_{1}(t)$ are time-dependent covariates and given by $W_{1}(t)=B_{11} I\left(t \leq V_{1}\right)+B_{12} I\left(t>V_{1}\right)$ and $Z_{1}(t)=B_{21} I\left(t \leq V_{2}\right)+B_{22} I\left(t>V_{2}\right)$, where $B_{j 1}, B_{j 2}$ and $V_{j}$ were generated independently from the uniform distributions over $(0,0.5),(0.5,1)$ and $(0, \tau)$ with $\tau=1$, respectively, $j=1,2$. Furthermore, $W_{2}(t)$ and $Z_{2}(t)$ were assumed to be timeindependent and generated from the uniform distribution over $(0,1)$ independently.

Note that the covariates generated above are mutually independent. Corresponding to this, we also considered the situation where they are dependent. For this, we first generated $\left(L_{1}, \ldots, L_{6}\right)$ from the multivariate normal distribution with $E\left[L_{j}\right]=1$ for $j=1, \ldots, 6$ and $\operatorname{cov}\left(L_{j}, L_{k}\right)=0.25$ if $j \neq k$ and 1 if $j=k$ for $j, k=1, \ldots, 6$. Then we generated $W_{1}(t)$ and $Z_{1}(t)$ as above but setting $B_{11}=\Phi\left(L_{1}\right) / 2, B_{12}=$ $\left(\Phi\left(L_{1}\right)+\Phi\left(L_{2}\right)\right) / 2, B_{21}=\Phi\left(L_{3}\right) / 2, B_{22}=\left(\Phi\left(L_{3}\right)+\Phi\left(L_{4}\right)\right) / 2$ with $V_{1}$ and $V_{2}$ generated independently from the uniform distribution over $(0, \tau)$. In addition, we set $W_{2}=\Phi\left(L_{5}\right) / 2, Z_{2}=\Phi\left(L_{6}\right) / 2$. For the regression parameters, we set $\gamma_{1}=0.5, \gamma_{2}=0.5$ and considered two set-ups for $\beta(t)$ :

Scenario $1 \beta_{1}(t)=t$ and $\beta_{2}(t)=t^{2}$

Scenario $2 \beta_{1}(t)=(\sin (4 \pi t)+4 \pi t) / 12$ and $\beta_{2}(t)=(\cos (4 \pi t)+4 \pi t) / 12$.

To generate the panel count data, we first generated the observation times $t_{i, j}$ 's from the non-homogeneous Poisson process with the mean function $3 t+4$ and the 
follow-up times $C_{i}$ 's following the uniform distribution over $(0.9 \tau, \tau)$. Then, given the covariates and the real observation times, the panel count data were generated by $N_{i}\left(t_{i, j}\right)=\sum_{k=1}^{j} N_{i}^{*}\left(t_{i, k}\right)-N_{i}^{*}\left(t_{i, k-1}\right)$ with $t_{i, 0}=0$, where $N_{i}^{*}\left(t_{i, k}\right)-N_{i}^{*}\left(t_{i, k-1}\right)$ follows the Poisson distribution with the mean

$$
v_{i} \Lambda_{0}\left(t_{i, k}\right) e^{\left(\boldsymbol{\gamma}^{T} \mathbf{W}_{i}\left(t_{i, k}\right)+\boldsymbol{\beta}^{T}\left(t_{i, j}\right) \mathbf{Z}_{i}\left(t_{i, j}\right)\right)}-v_{i} \Lambda_{0}\left(t_{i, j-1}\right) e^{\left(\boldsymbol{\gamma}^{T} \mathbf{W}_{i}\left(t_{i, j-1}\right)+\boldsymbol{\beta}^{T}\left(t_{i, j-1}\right) \mathbf{Z}_{i}\left(t_{i, j-1}\right)\right)}
$$

given the $v_{i}$ 's. Here we set $\Lambda_{0}(t)=2 t+3$ or $\Lambda_{0}(t)=(\sin (4 \pi t)+4 \pi t) / 2$ and considered two settings for the $v_{i}$ 's. One is that $v_{i}=1$ for all $i$, meaning that the $N_{i}$ 's are Poisson processes with the mean function $\Lambda_{0}(t) \exp \left\{\boldsymbol{\gamma}^{T} \mathbf{W}_{i}(t)+\boldsymbol{\beta}^{T}(t) \mathbf{Z}_{i}(t)\right\}$, and the other is that $v_{i}$ follows the gamma distribution with mean 1 and variance $\sigma^{2}=1$, meaning that the $N_{i}(t)$ 's are mixed Poisson processes with the same mean function as above. The results given below are based on $n=300$ with 1000 replications.

Table 1 presents the results on estimation of two time-independent regression coefficients $\gamma_{1}$ and $\gamma_{2}$ with $\Lambda_{0}(t)=2 t+3$. Here we used the cubic B-splines with three internal knots equally spaced on $(0, \tau)$. The results include the empirical bias (BIAS) given by the average of the estimates minus the true value, the sampling standard deviation (SE), the average of the estimated standard errors (ESE), and the $95 \%$ empirical coverage probability (CP). Note that for the variance estimation, in addition to the robust estimation discussed above, we also applied the simple 
bootstrap procedure with 200 bootstrapped samples for comparison. They suggest that the proposed estimators seem to be unbiased and the variance estimations also appear to be appropriate. In addition, the results on $\mathrm{CP}$ indicate that the normal approximation to the distribution of the proposed estimator $\hat{\gamma}$ seems to be reasonable too.

Figure 1 gives the averages of the estimated $\beta_{1}(t)$ and $\beta_{2}(t)$ obtained under the situation where the $N_{i}(t)$ 's are Poisson processes with independent covariates over 1000 equal-spaced grid points on the time axis. For comparison, the true function is also included in the figure, and one can see that the proposed procedure seems to yield unbiased estimates again. Furthermore, Figure 1 also shows the respective average of the estimated pointwise standard errors by the robust and bootstrap methods along with the pointwise empirical standard errors of $\boldsymbol{\beta}(t)$. Again they indicate that the proposed method appears to give reasonable variance estimates. The same can be seen from Figure 2, which provides the results on estimation of $\beta_{1}(t)$ and $\beta_{2}(t)$ as Figure 1 except under the case of the mixed Poisson process with independent covariates.

Table 2 provides the results on estimation of two time-independent regression coefficients $\gamma_{1}$ and $\gamma_{2}$ obtained under the same set-up as Table 1 except $\Lambda_{0}(t)=$ $(\sin (4 \pi t)+4 \pi t) / 2$. The corresponding results on estimation of $\beta_{1}(t)$ and $\beta_{2}(t)$ are given in Figures 3-4. It is apparent that they gave similar conclusions as above, and 
the same is true for the situation of dependent covariates, for which the results are provided in the Supplementary Material due to the limited space. We also considered other set-ups, including different degrees of B-spline functions, different numbers of interior knots and other types of covariates, and obtained similar results.

Table 1: Simulation results on estimation of $\gamma_{1}$ and $\gamma_{2}$ with $\Lambda_{0}(t)=2 t+3$.

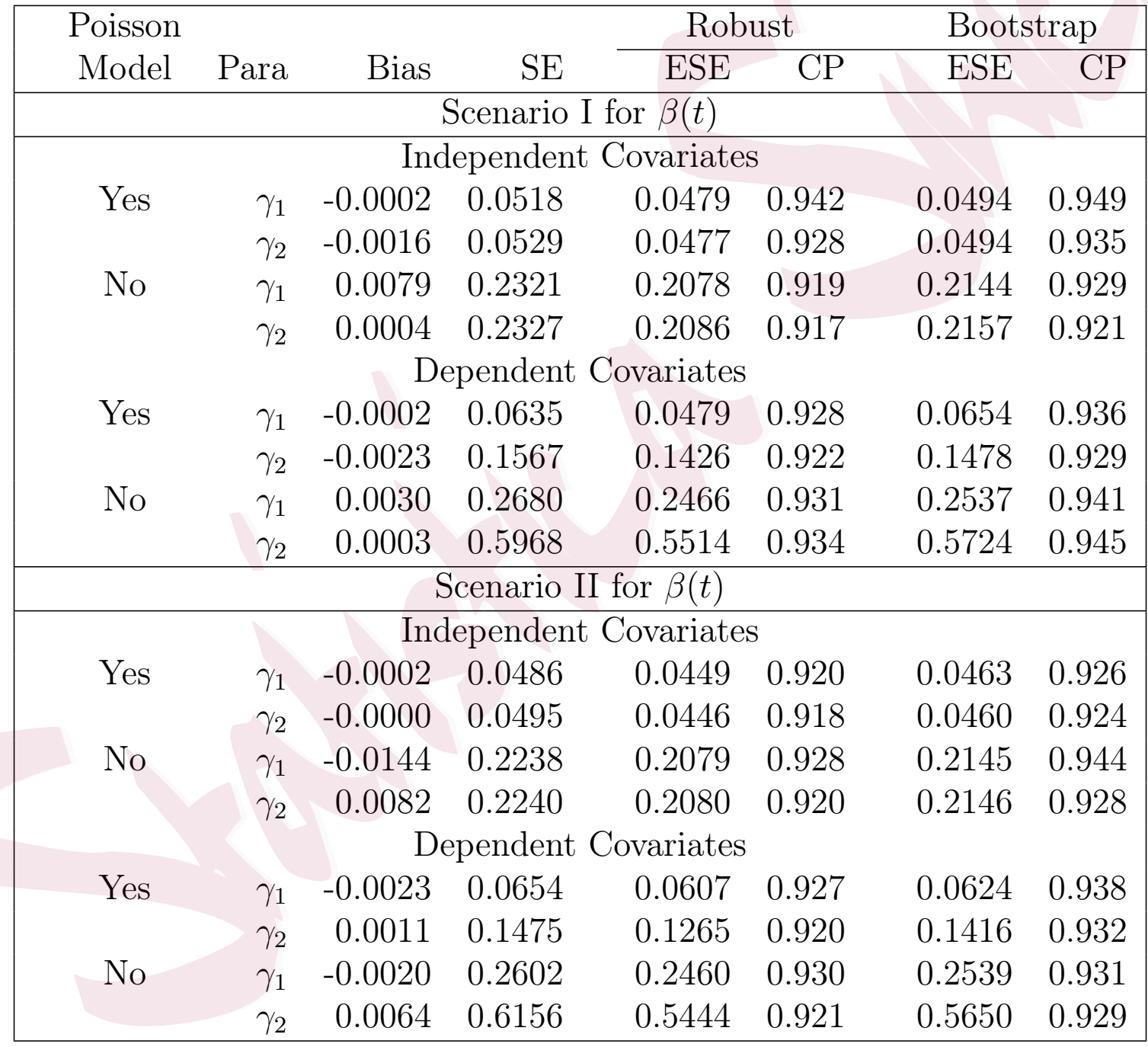


Table 2: Simulation results on estimation of $\gamma_{1}$ and $\gamma_{2}$ with $\Lambda_{0}(t)=(\sin (4 \pi t)+4 \pi t) / 2$.

\begin{tabular}{|c|c|c|c|c|c|c|c|}
\hline \multirow{2}{*}{$\begin{array}{c}\text { Poisson } \\
\text { Model }\end{array}$} & \multirow[b]{2}{*}{ Para } & \multirow[b]{2}{*}{ Bias } & \multirow[b]{2}{*}{$\mathrm{SE}$} & \multicolumn{2}{|c|}{ Robust } & \multicolumn{2}{|c|}{ Bootstrap } \\
\hline & & & & $\overline{\mathrm{ESE}}$ & $\mathrm{CP}$ & ESE & $\mathrm{CP}$ \\
\hline \multicolumn{8}{|c|}{ Scenario I for $\beta(t)$} \\
\hline \multicolumn{8}{|c|}{ Independent Covariates } \\
\hline \multirow[t]{2}{*}{ Yes } & $\gamma_{1}$ & -0.0009 & 0.0521 & 0.0485 & 0.933 & 0.0501 & 0.941 \\
\hline & $\gamma_{2}$ & 0.0012 & 0.0531 & 0.0485 & 0.925 & 0.0501 & 0.929 \\
\hline \multirow[t]{3}{*}{ No } & $\gamma_{1}$ & -0.0023 & 0.2484 & 0.2217 & 0.919 & 0.2289 & 0.928 \\
\hline & $\gamma_{2}$ & -0.0028 & 0.2508 & 0.2212 & 0.926 & 0.2291 & 0.932 \\
\hline & \multicolumn{7}{|c|}{ Dependent Covariates } \\
\hline \multirow[t]{2}{*}{ Yes } & $\gamma_{1}$ & 0.0016 & 0.0659 & 0.0631 & 0.931 & 0.0651 & 0.939 \\
\hline & $\gamma_{2}$ & 0.0013 & 0.1490 & 0.1372 & 0.923 & 0.1420 & 0.936 \\
\hline \multirow[t]{2}{*}{ No } & $\gamma_{1}$ & 0.0035 & 0.2766 & 0.2573 & 0.928 & 0.2659 & 0.938 \\
\hline & $\gamma_{2}$ & 0.0027 & 0.6337 & 0.5610 & 0.916 & 0.5852 & 0.927 \\
\hline \multicolumn{8}{|c|}{ Scenario II for $\beta(t)$} \\
\hline \multicolumn{8}{|c|}{ Independent Covariates } \\
\hline \multirow[t]{2}{*}{ Yes } & $\gamma_{1}$ & -0.0002 & 0.0476 & 0.0449 & 0.931 & 0.0501 & 0.950 \\
\hline & $\gamma_{2}$ & -0.0001 & 0.0489 & 0.0449 & 0.922 & 0.0501 & 0.943 \\
\hline \multirow[t]{3}{*}{ No } & $\gamma_{1}$ & 0.0104 & 0.2505 & 0.2199 & 0.918 & 0.2270 & 0.920 \\
\hline & $\gamma_{2}$ & -0.0157 & 0.2442 & 0.2200 & 0.921 & 0.2273 & 0.923 \\
\hline & & & pendent & ovariates & & & \\
\hline \multirow[t]{2}{*}{ Yes } & $\gamma_{1}$ & 0.0009 & 0.0662 & 0.0608 & 0.929 & 0.0628 & 0.932 \\
\hline & $\gamma_{2}$ & -0.0005 & 0.1436 & 0.1344 & 0.942 & 0.1390 & 0.941 \\
\hline \multirow[t]{2}{*}{ No } & $\gamma_{1}$ & -0.0009 & 0.2750 & 0.2559 & 0.930 & 0.2641 & 0.940 \\
\hline & $\gamma_{2}$ & -0.0166 & 0.6369 & 0.5594 & 0.923 & 0.5825 & 0.930 \\
\hline
\end{tabular}


(a1) Scenario 1: $\beta_{1}(\mathrm{t})$ point estimation

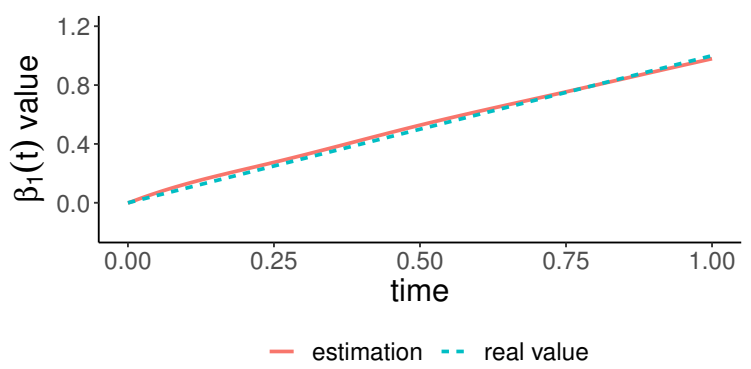

(a3) Scenario 2: $\beta_{1}(\mathrm{t})$ point estimation

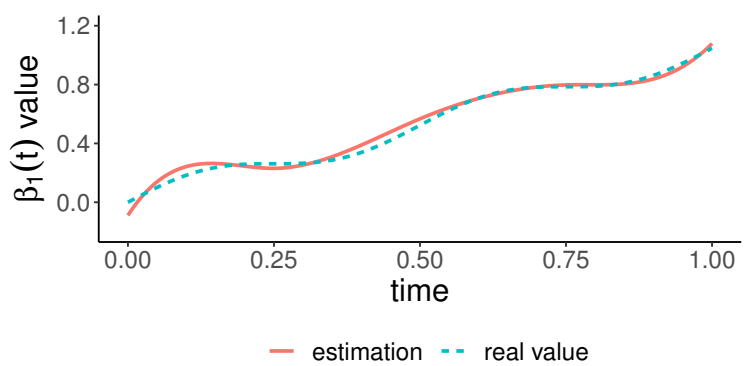

(a5) Scenario 1: $\beta_{1}(\mathrm{t})$ variance estimation

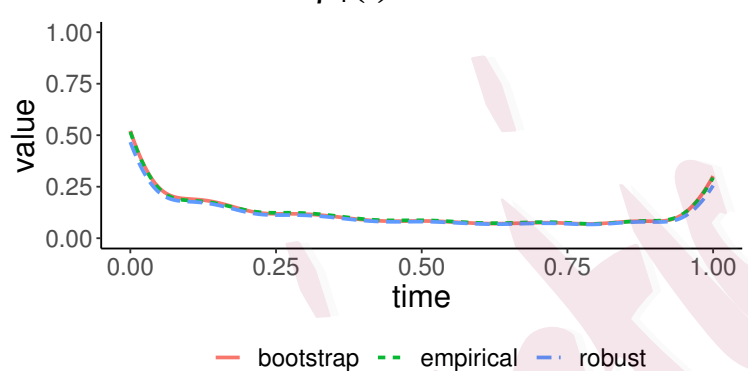

(a7) Scenario 2: $\beta_{1}(t)$ variance estimation

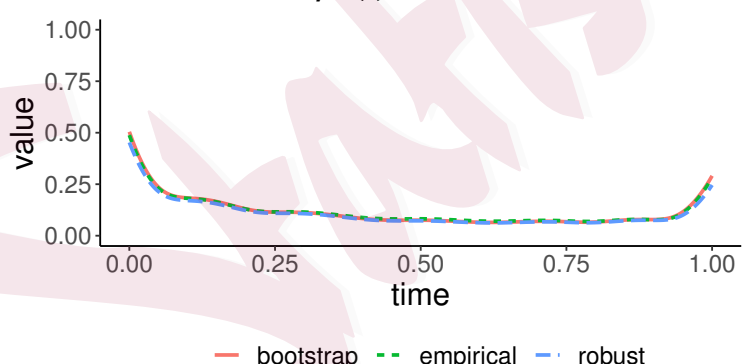

(a2) Scenario 1: $\beta_{2}(\mathrm{t})$ point estimation

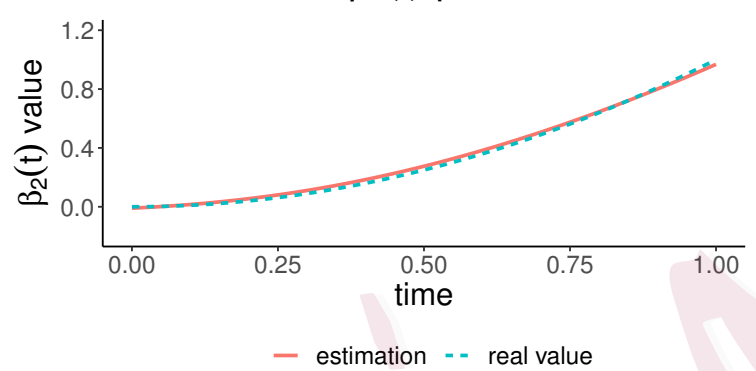

(a4) Scenario 2: $\beta_{2}(t)$ point estimation

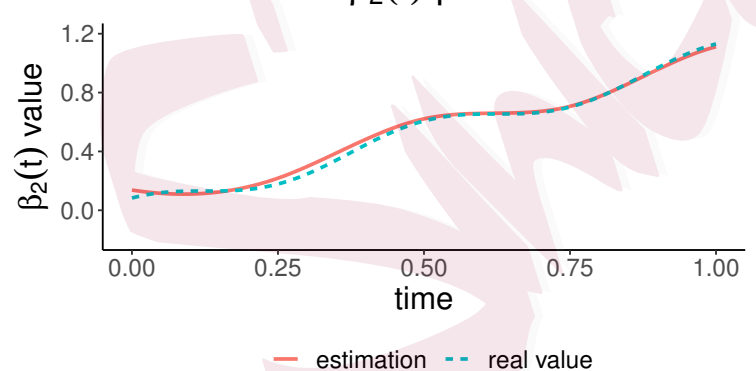

(a6) Scenario 1: $\beta_{2}(\mathrm{t})$ variance estimation

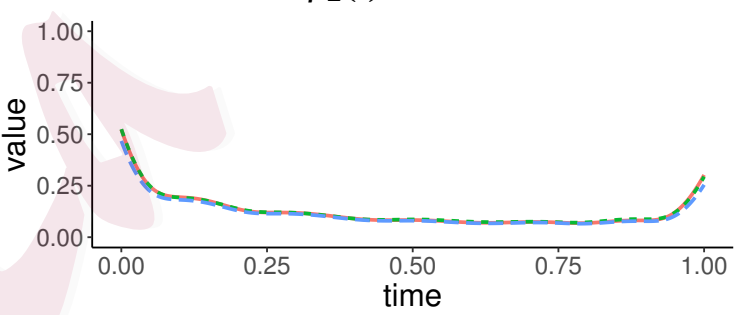

- bootstrap - - empirical - robust

(a8) Scenario 2: $\beta_{2}(\mathrm{t})$ variance estimation

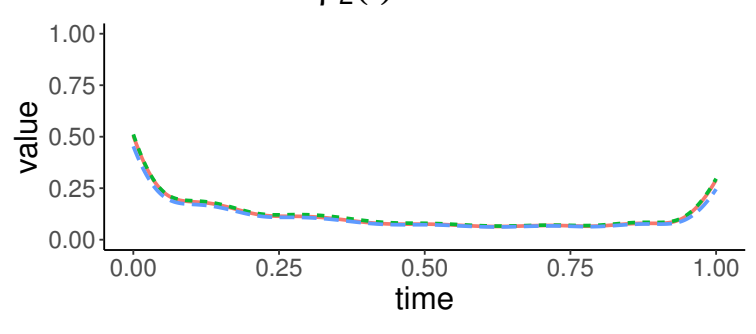

- bootstrap =- empirical - ' robust

Figure 1: Simulation results on estimation of $\beta_{1}(t)$ and $\beta_{2}(t)$ under the Poisson process with $\Lambda_{0}(t)=2 t+3$ and independent covariates. (a1) - (a4): on estimation of $\beta_{1}(t)$ and $\beta_{2}(t)$; (a5) - (a8): on variance estimation of $\hat{\beta}_{1}(t)$ and $\hat{\beta}_{2}(t)$. 
(a1) Scenario 1: $\beta_{1}(\mathrm{t})$ point estimation

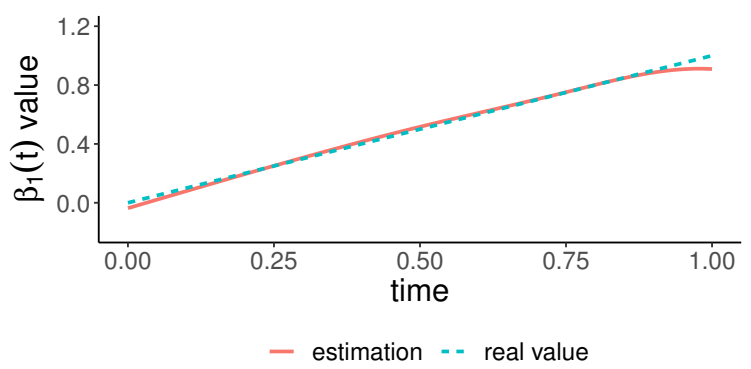

(a3) Scenario 2: $\beta_{1}(\mathrm{t})$ point estimation

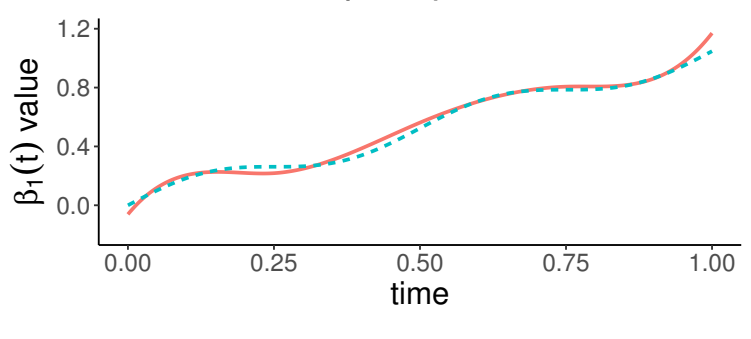

- estimation =- real value

(a5) Scenario 1: $\beta_{1}(\mathrm{t})$ variance estimation

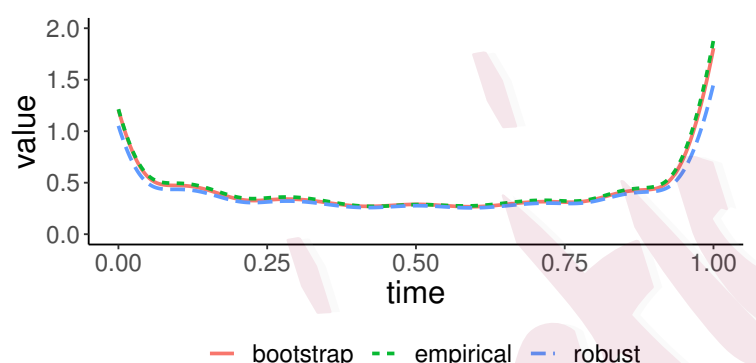

(a7) Scenario 2: $\beta_{1}(\mathrm{t})$ variance estimation

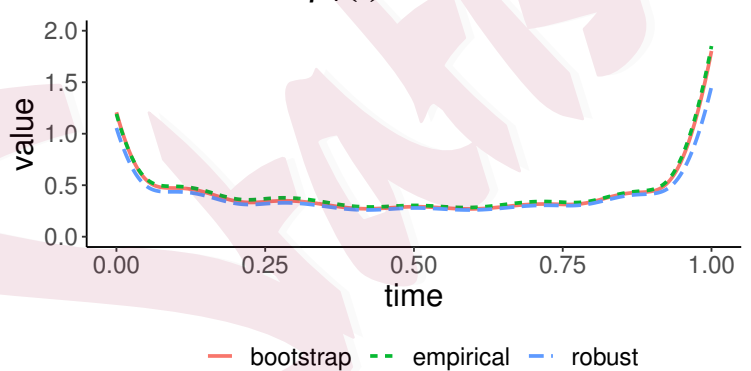

(a2) Scenario 1: $\beta_{2}(\mathrm{t})$ point estimation

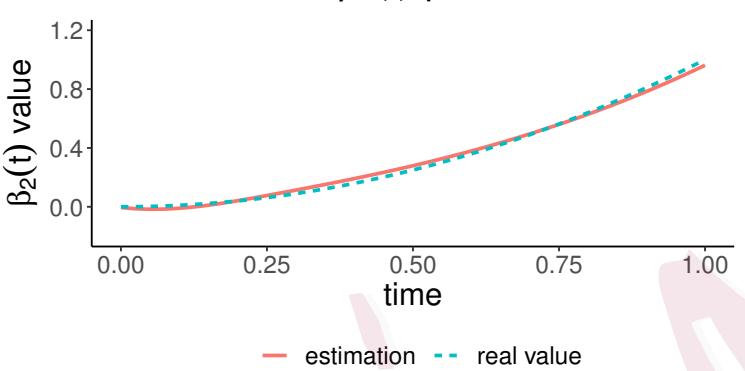

(a4) Scenario 2: $\beta_{2}(t)$ point estimation

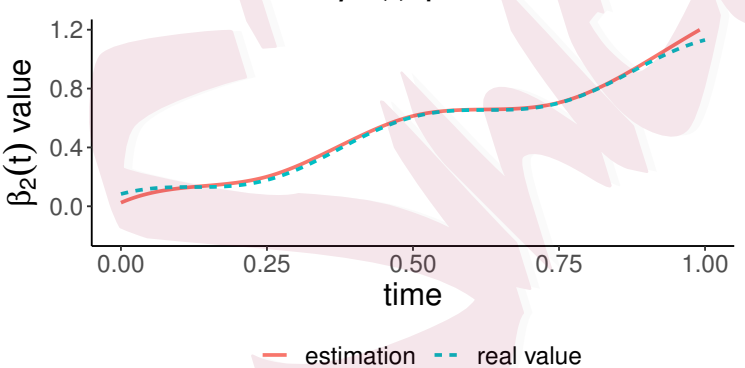

(a6) Scenario 1: $\beta_{2}(t)$ variance estimation

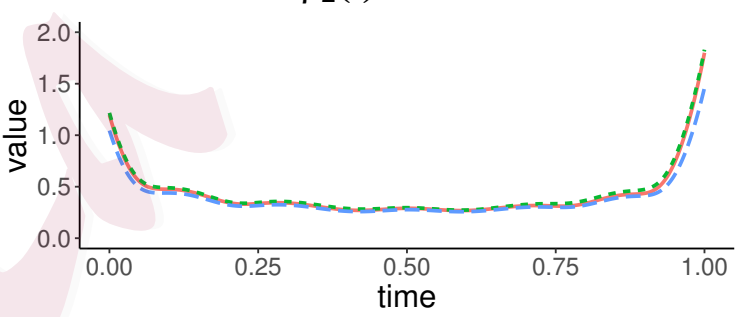

- bootstrap -- empirical - robust

(a8) Scenario 2: $\beta_{2}(t)$ variance estimation

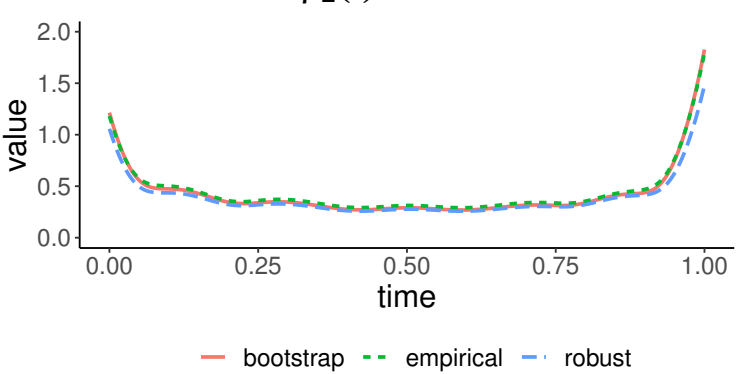

Figure 2: Simulation results on estimation of $\beta_{1}(t)$ and $\beta_{2}(t)$ under the Non-Poisson process with $\Lambda_{0}(t)=2 t+3$ and independent covariates. (a1) - (a4): on estimation of $\beta_{1}(t)$ and $\beta_{2}(t)$; (a5) - (a8): on variance estimation of $\hat{\beta}_{1}(t)$ and $\hat{\beta}_{2}(t)$. 
(a1) Scenario 1: $\beta_{1}(\mathrm{t})$ point estimation

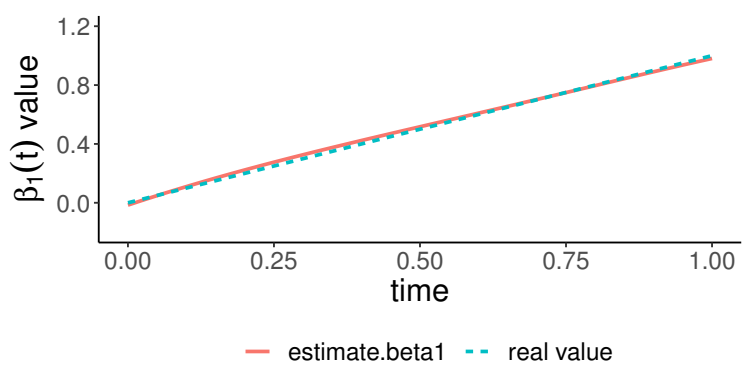

(a3) Scenario 2: $\beta_{1}(\mathrm{t})$ point estimation

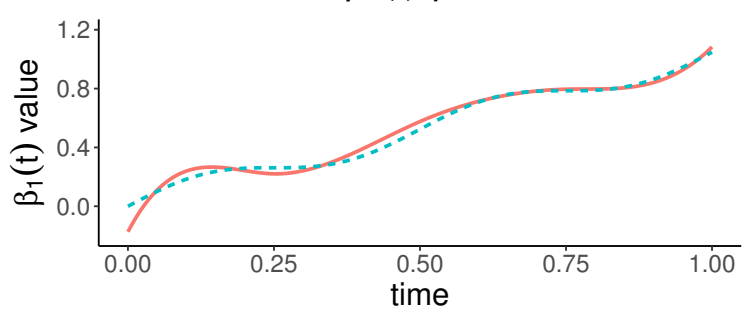

- estimation =- real value

(a5) Scenario 1: $\beta_{1}(\mathrm{t})$ variance estimation

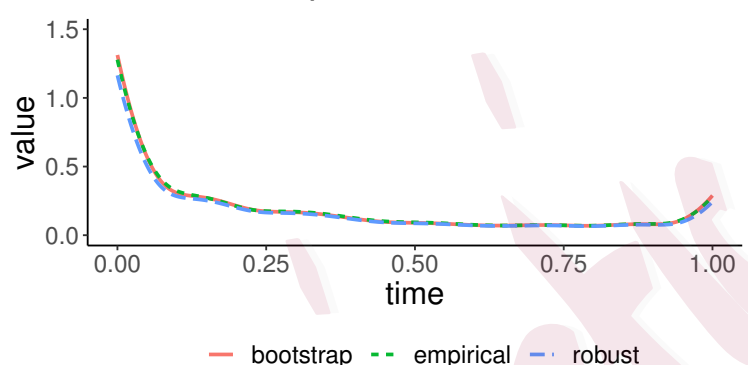

(a7) Scenario 2: $\beta_{1}(\mathrm{t})$ variance estimation

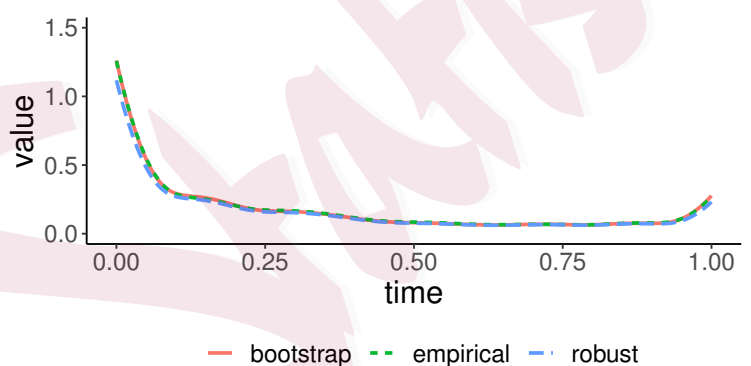

(a2) Scenario 1: $\beta_{2}(\mathrm{t})$ point estimation

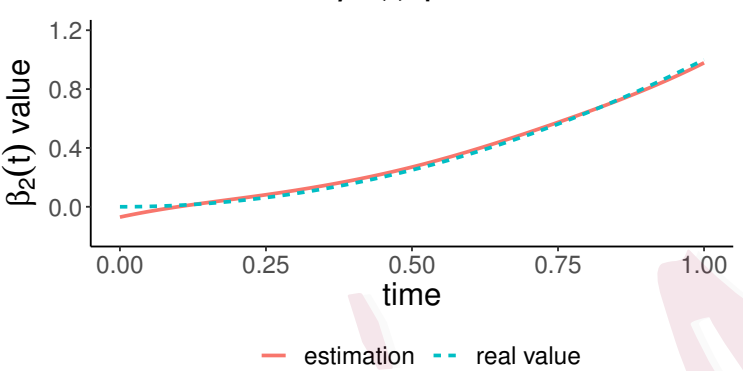

(a4) Scenario 2: $\beta_{2}(t)$ point estimation

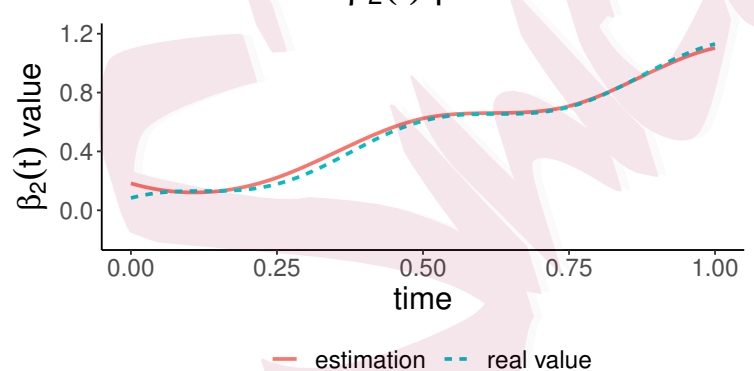

(a6) Scenario 1: $\beta_{2}(t)$ variance estimation

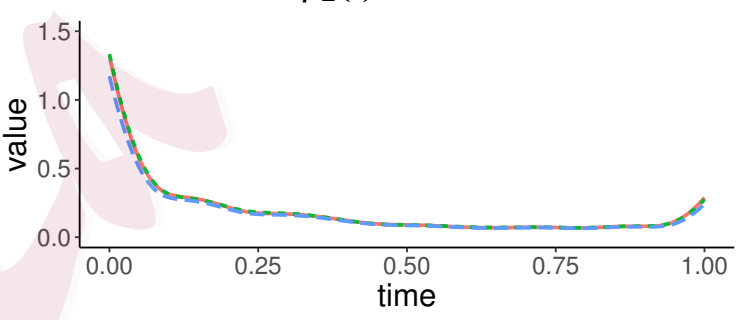

- bootstrap -- empirical - robust

(a8) Scenario 2: $\beta_{2}(\mathrm{t})$ variance estimation

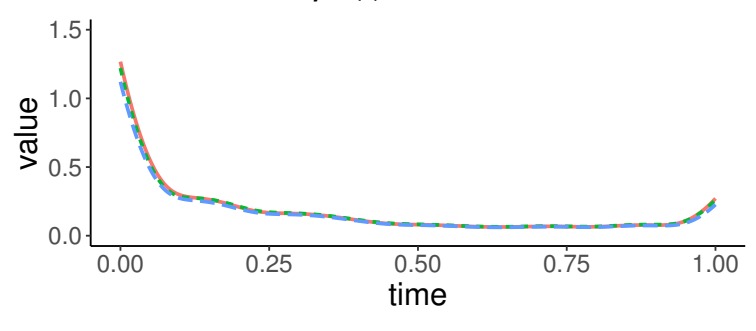

- bootstrap - = empirical - robust

Figure 3: Simulation results on estimation of $\beta_{1}(t)$ and $\beta_{2}(t)$ under the Poisson process with $\Lambda_{0}(t)=(\sin (4 \pi t)+4 \pi t) / 2$ and independent covariates. (a1) - (a4): on estimation of $\beta_{1}(t)$ and $\beta_{2}(t)$; (a5) - (a8): on variance estimation of $\hat{\beta}_{1}(t)$ and $\hat{\beta}_{2}(t)$. 
(a1) Scenario 1: $\beta_{1}(\mathrm{t})$ point estimation

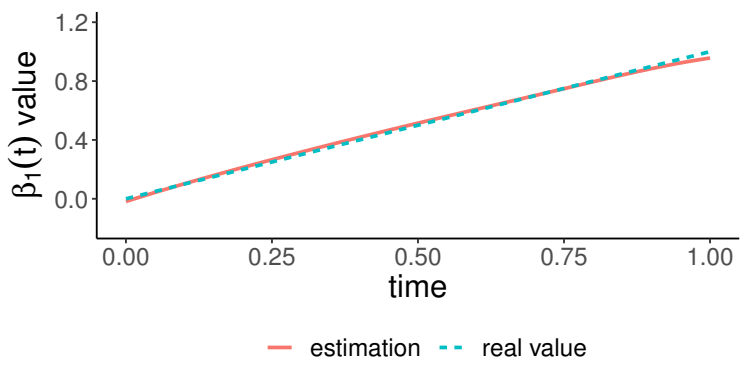

(a3) Scenario 2: $\beta_{1}(\mathrm{t})$ point estimation

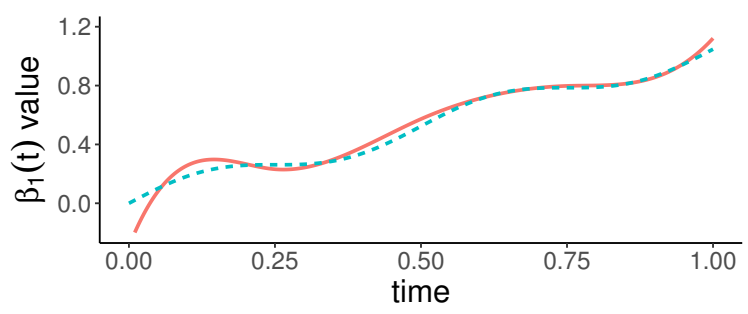

- estimation $=-$ real value

(a5) Scenario 1: $\beta_{1}(\mathrm{t})$ variance estimation

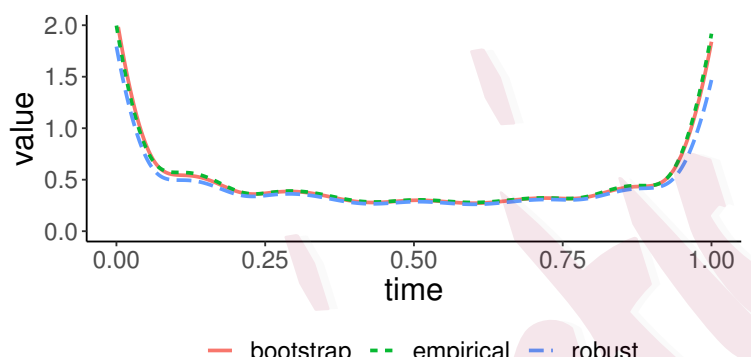

(a7) Scenario 2: $\beta_{1}(\mathrm{t})$ variance estimation

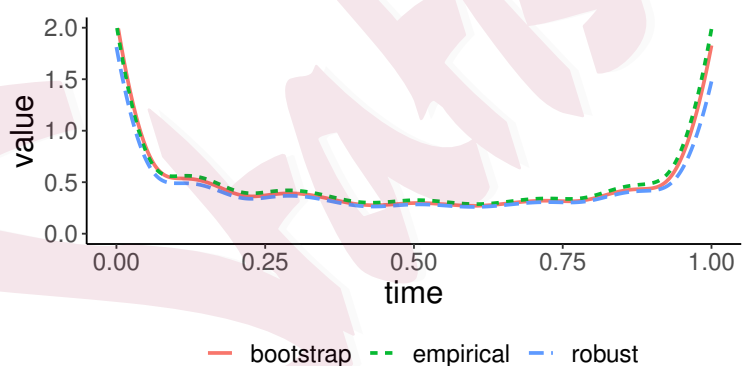

(a2) Scenario 1: $\beta_{2}(\mathrm{t})$ point estimation

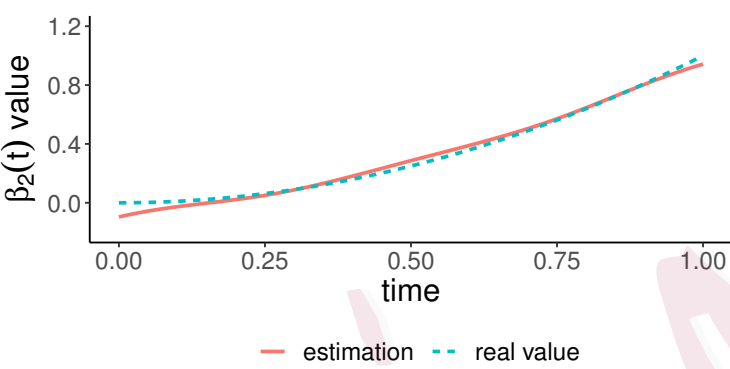

(a4) Scenario 2: $\beta_{2}(\mathrm{t})$ point estimation

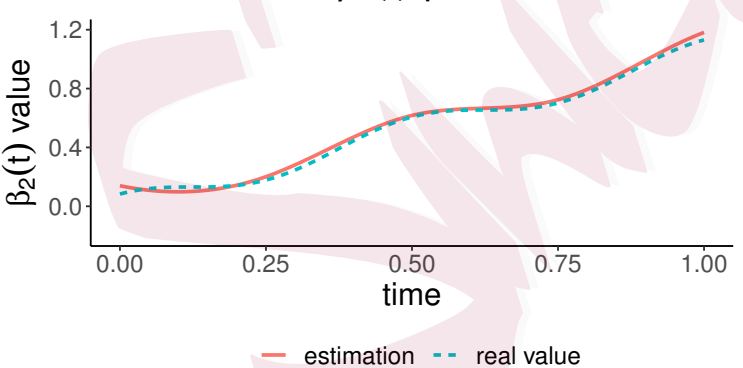

(a6) Scenario 1: $\beta_{2}(t)$ variance estimation

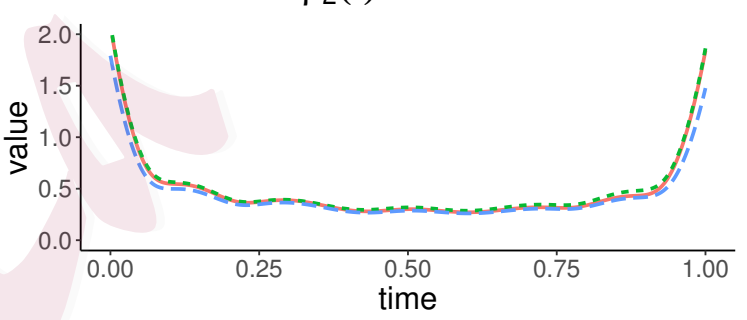

- bootstrap - - empirical - robust

(a8) Scenario 2: $\beta_{2}(t)$ variance estimation

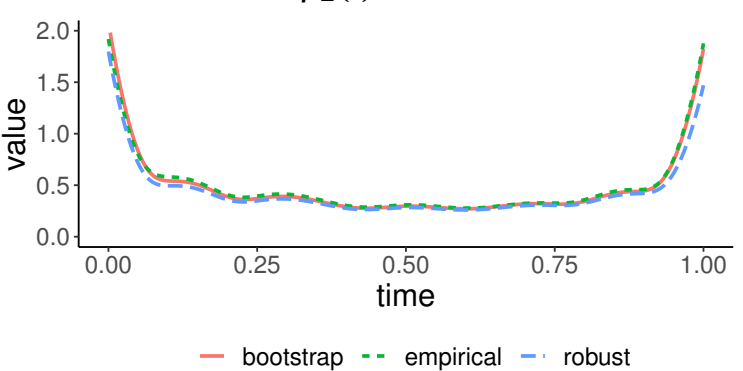

Figure 4: Simulation results on estimation of $\beta_{1}(t)$ and $\beta_{2}(t)$ under the Non-Poisson process with $\Lambda_{0}(t)=(\sin (4 \pi t)+4 \pi t) / 2$ and independent covariates. (a1) - (a4): on estimation of $\beta_{1}(t)$ and $\beta_{2}(t)$; (a5) - (a8): on variance estimation of $\hat{\beta}_{1}(t)$ and $\hat{\beta}_{2}(t)$. 


\section{Analysis of China Health and Nutrition Survey}

Now we apply the methodology proposed in the previous sections to the China Health and Nutrition Survey (CHNS) discussed above. It is an international collaborative project between the Carolina Population Center at the University of North Carolina at Chapel Hill and the National Institute for Nutrition and Health at the Chinese Center for Disease Control and Prevention. The survey took place over a 7-day period using a multistage, random cluster process to draw a sample of over 11000 households with over 42,000 individuals participated in 15 provinces and municipal cities that vary substantially in geography, economic development, public resources, and health indicators. Villages and townships within the counties and urban/suburban neighborhoods within the cities were selected randomly.

As mentioned before, among others, one objective of interest is to assess the relationship between the pregnancy process of the female participants and various factors. Also as discussed above, due to the nature of the study, only panel count data are available for the pregnancy occurrence process. For the analysis below, we will focus on the 2537 female participants with complete information on the following four covariates: whether the mother came from urban or rural areas (0: urban, 1: rural), the average monthly wage last year, the completed formal education level in regular school (0: No school; 1: Primary school; 2: Middle school; 3: Technical school; 4: College)), and the current health status (1: Excellent, 2: Good, 3: Fair, 
4: Poor). Table 3 gives a descriptive summary of the four covariates at the baseline and the range of the pregnancies is from one to eight.

For the application of the proposed estimation procedure, we first assume that all of the four covariates had time-varying effects and Figure 5 presents the estimated covariate effects with 3 interior knots for the B-splines. The results suggest that the mother's location seems to have a significantly positive relationship with the fertility occurrence rate and the effect magnitude appears to increase along with the mothers' age. In other words, the mothers from rural areas were more likely to have more children compared to those from urban areas, and the differences in the number of pregnancy increased with the women getting older. In contrast, the mother's education level seems to have significantly negative effects on the number of pregnancy, indicating that well-educated mothers tended to have fewer children and the effect increased as the women became older. Furthermore, it seems that both the average monthly wage and the mother's health status had no significant time-varying effects or might only have constant effects on the pregnancy process.

To further investigate the estimated effects above, following a suggestion from a referee, we repeat the analysis above by adding the interaction effect between the location and education level and the results are presented in Figure 6. One can see that it gives similar results on four individual factors and suggests that there seems to exist significantly negative interaction effects for mid-aged mothers. In 
other words, it indicates that the education level effect magnitude increased faster for female subjects in rural areas. Note that one may not want to pay much attention to the estimated effects after age 60 due to the sparsity of the observed data.

Based on the results above, we again apply the proposed estimation procedure but assume that only the location, the education level and their interaction may have time-varying effects. In other words, it is supposed that both the average monthly wage and the health status only had constant effects on the pregnancy process. Table 4 gives the estimated covariate effects for the average monthly wage and current health status with 3,5 or 7 interior knots for the B-splines. The estimated timedependent effects based on 3 interior knots are presented in Figure 7, while the results with 5 or 7 interior knots are similar and provided in the Supplementary Material. One can see from Table 4 that the mothers' average monthly wage was significantly negatively correlated with the fertility rate and the mothers with lower income tended to have more children. In contrast, the health status level had positive effects on the fertility rate, and all results are consistent with respect to the number of interior knots. Finally the results given in Figure 7 are apparently similar to those given in Figure 6 and again indicate the existence of the time-varying effect and the necessity of the use of the proposed method. 
Table 3: Summary of the four covariates at the baseline for the CHNS.

\begin{tabular}{|cccc|}
\hline Covariate & Mean \pm SD & Median & Range \\
\hline $\begin{array}{c}\text { Average Monthly Wage } \\
\text { Last Year (Yuan) }\end{array}$ & $1635.13 \pm 3741.61$ & 800 & $8-99999$ \\
\hline \multirow{2}{*}{ Location } & Category & Count & Percentage \\
& Rural & 1366 & $53.84 \%$ \\
Education Level & Urban & 1171 & $46.16 \%$ \\
& 0: No school & 89 & $3.51 \%$ \\
& 1: Primary school & 359 & $14.15 \%$ \\
& 2: Middle school & 1373 & $54.12 \%$ \\
& 3: Technical school & 330 & $13.01 \%$ \\
& 4: College & 386 & $15.21 \%$ \\
& & & \\
Current Health Status & 1:Excellent & 410 & $16.16 \%$ \\
& 2:Good & 1351 & $54.25 \%$ \\
& 3:Fair & 713 & $28.10 \%$ \\
& 4:Poor & 63 & $2.48 \%$ \\
\hline
\end{tabular}

Table 4: The estimated time-constant effects of the average monthly wage (wage) and the current health status (health) for the CHNS.

\begin{tabular}{|ccccc|}
\hline \# of interior knots & Covariate & Estimated effect & SD & $95 \%$ CI \\
\hline 3 & wage & -0.0301 & 0.0152 & $(-0.0598,-0.0003)$ \\
& health & 0.0287 & 0.0111 & $(0.0069,0.0505)$ \\
\hline 5 & wage & -0.0300 & 0.0150 & $(-0.0595,-0.0004)$ \\
& health & 0.0292 & 0.0111 & $(0.0074,0.0510)$ \\
\hline 7 & wage & -0.0300 & 0.0150 & $(-0.0590,-0.0004)$ \\
& health & 0.0290 & 0.0111 & $(0.0072,0.0508)$ \\
\hline
\end{tabular}



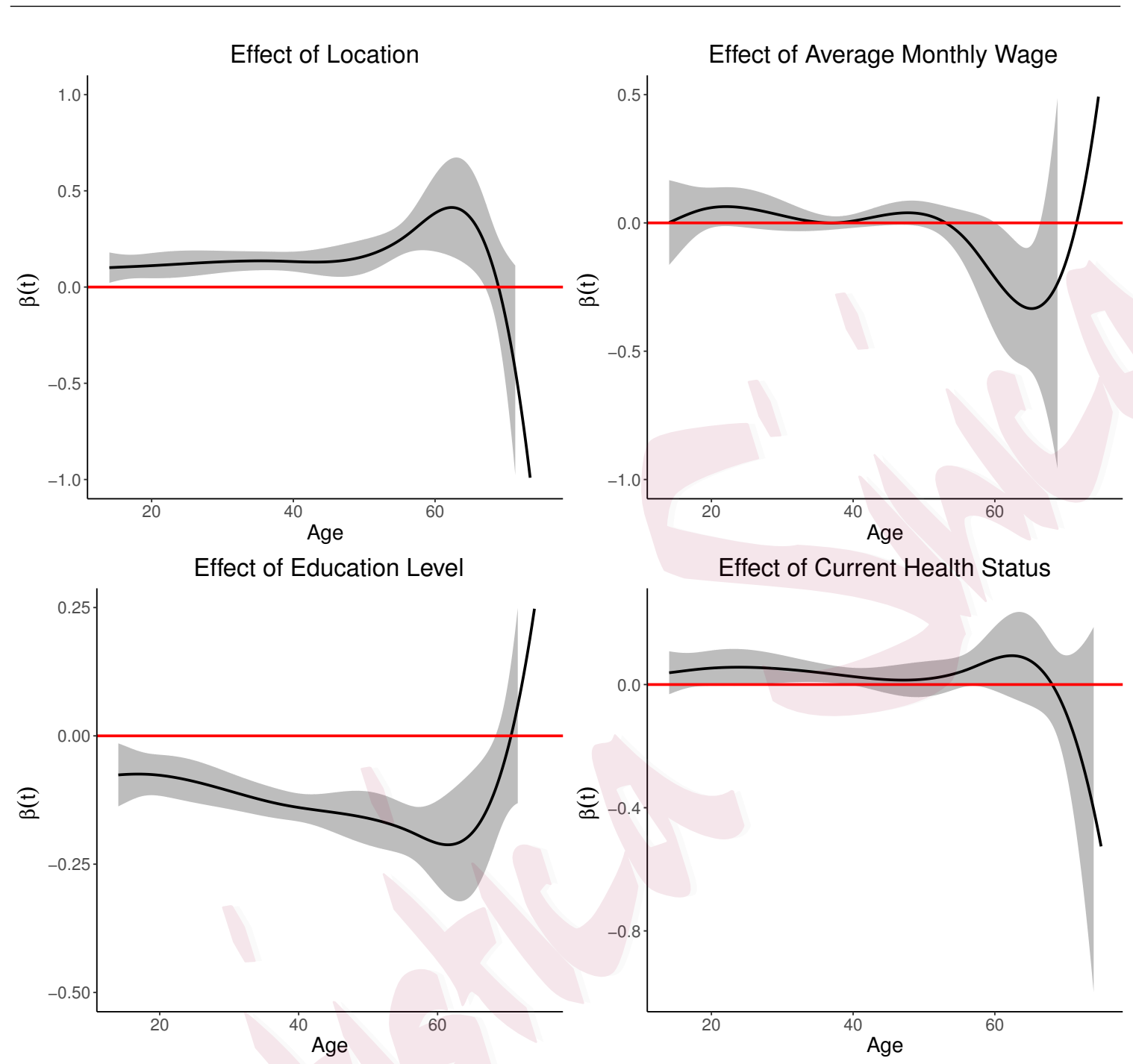

Figure 5: Estimated time-varying effects of all four covariates (solid curves) and corresponding pointwise $95 \%$ confidence intervals (gray ribbons). 

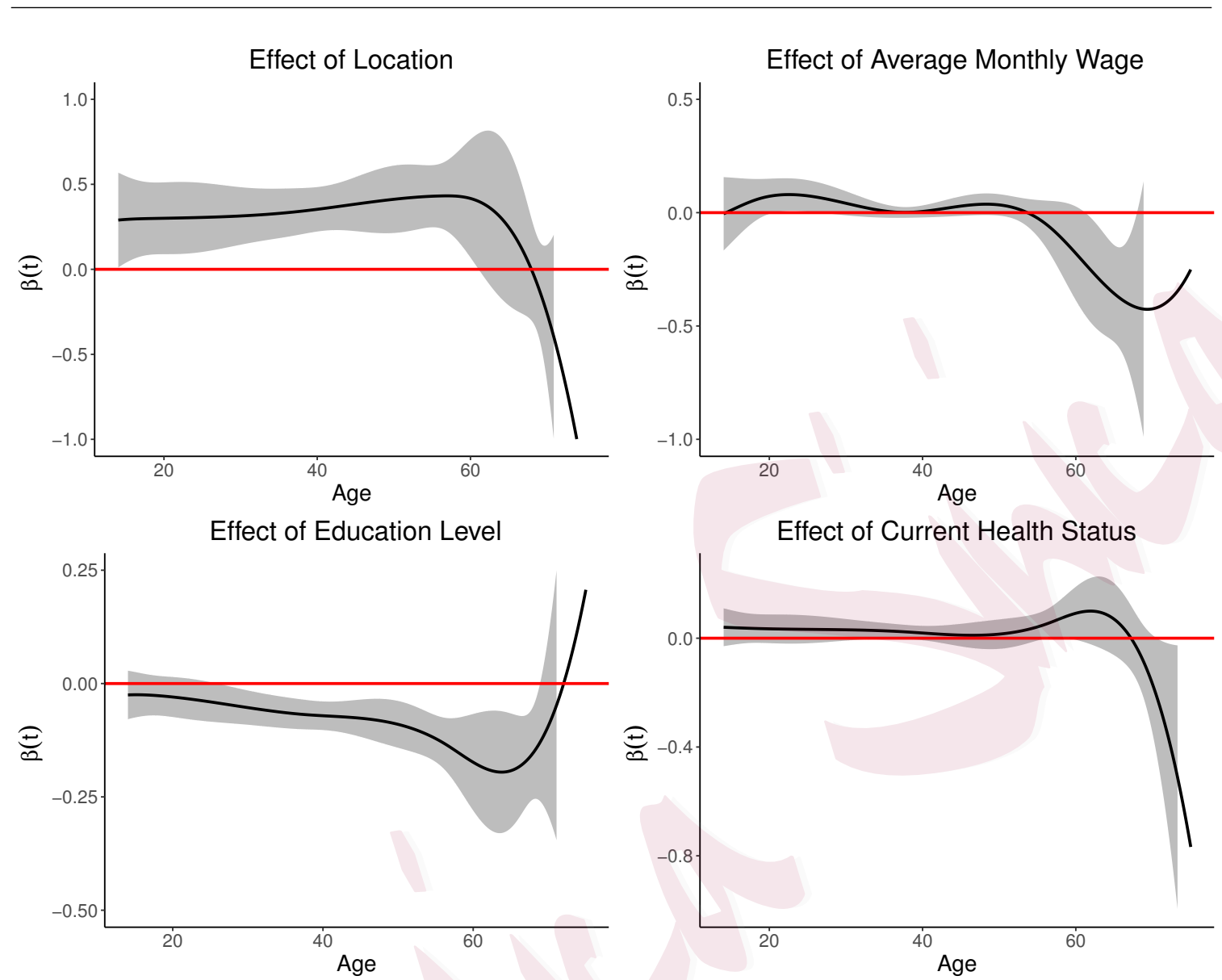

Effect of Interaction between Location and Education Level

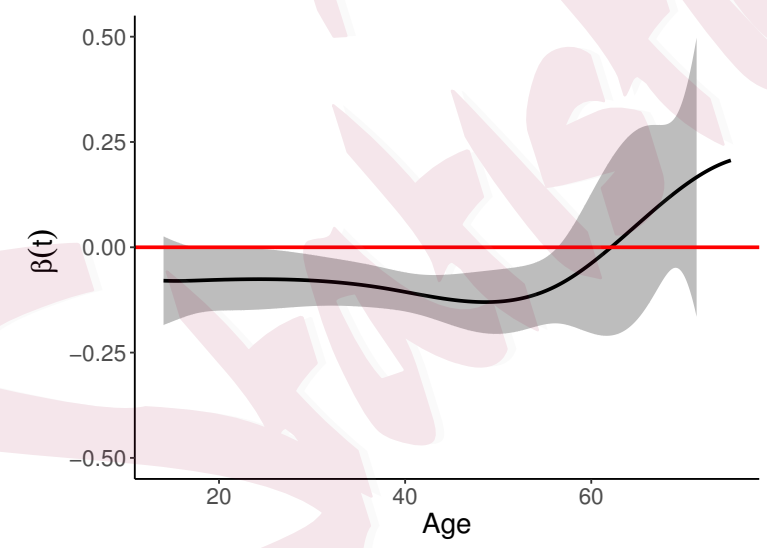

Figure 6: Estimated time-varying effects of all four covariates and the interaction (solid curves) and corresponding pointwise 95\% confidence intervals (gray ribbons). 

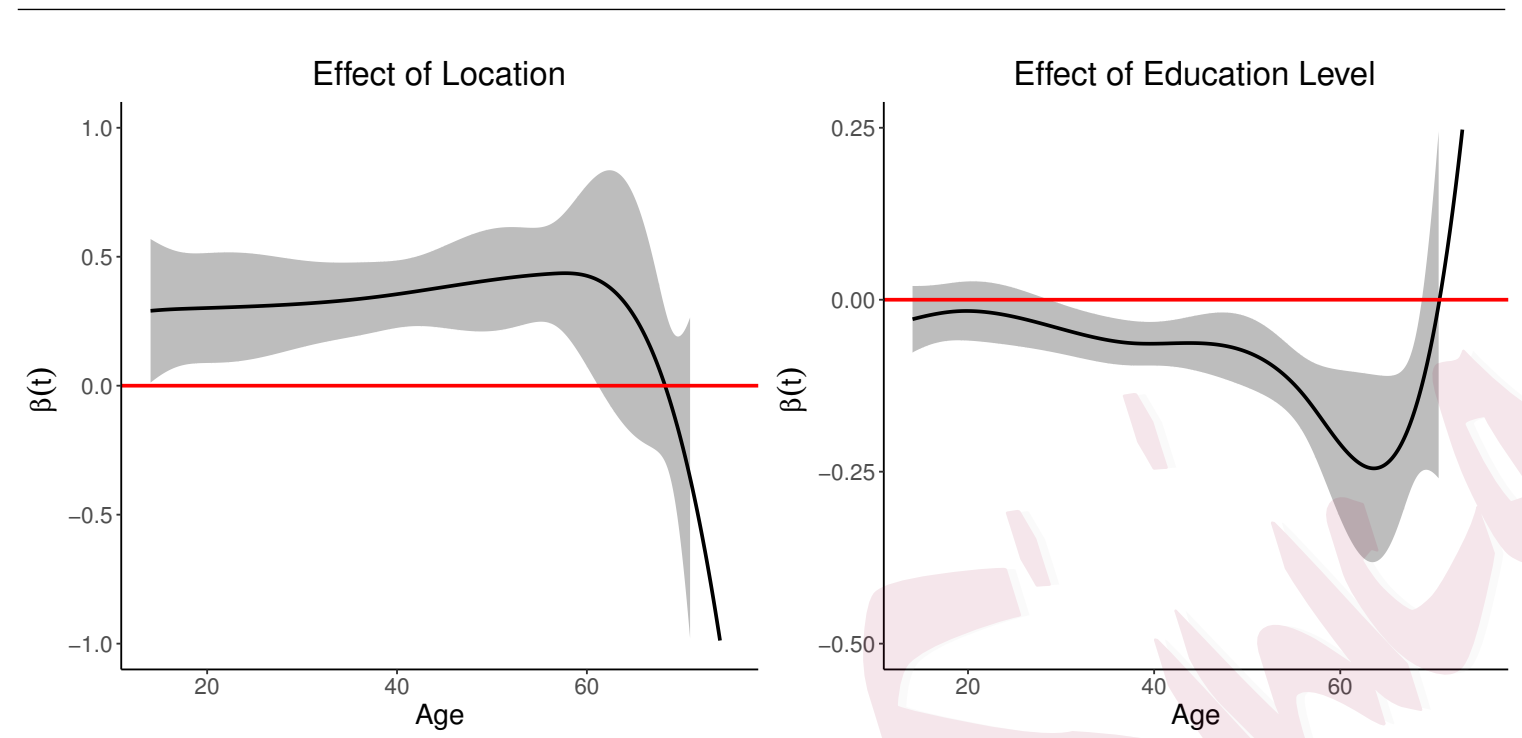

Effect of Interaction between Location and Education Level

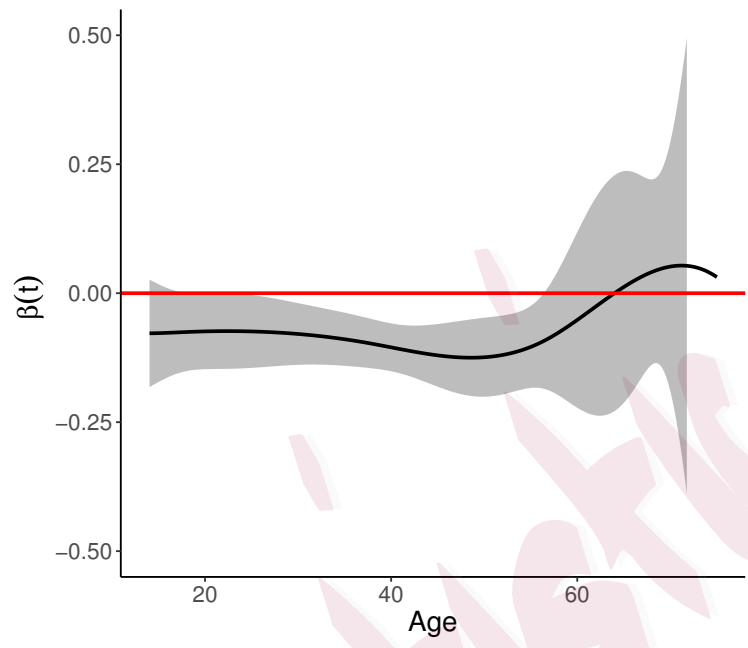

Figure 7: Estimated time-varying effects of the location, education level and the interaction between them (solid curves) and corresponding pointwise $95 \%$ confidence intervals (gray ribbons) with 3 interior knots. 


\section{Discussion and Concluding Remarks}

In this paper, we have discussed regression analysis of panel count data in the presence of both time-dependent covariates and time-varying covariate effects. For the problem, a spline-based estimating equation procedure was developed and the asymptotic properties of the proposed estimators were established. An extensive numerical study was conducted and suggested that the proposed method works well in practical situations. Also, the usefulness and necessity of the proposed estimation procedure was illustrated by its application to the CHNS, which identified some time-varying covariate effects.

There exist several directions for future research. One is that in the proposed method, we have assumed that the observation process does not depend on covariates and is also independent of the underlying recurrent event process of interest. In practice, it is apparent that this may not be true (Sun and Zhao, 2013). To develop a valid estimation procedure, one may need to model the three processes together. Another assumption used above is that the underlying recurrent event process follows the proportional mean model, meaning that the mean functions associated with any two sets of covariate values are proportional over time. Sometimes this may be too restrictive (Lin et al., 2001) and correspondingly, one may want to consider other models such as the class of semiparametric transformation mean models and develop some estimation procedures (Li et al., 2010). 
In the proposed estimation procedure, we also assumed that one knows which covariates have time-varying effects or time-constant effects. In practice, however, it is usually unknown and a simple approach is to try different combinations as shown in the application above. On the other hand, it would be useful to develop a data-driven procedure for the separation of the two types of covariates. In practice, one problem that one may often be interested in is the hypothesis testing about $\beta_{0}(\cdot)$. Theoretically, one could use the results given in Theorem 3 to develop a test procedure. On the other hand, this may not be straightforward since we cannot derive the explicit relationship between $\boldsymbol{h}_{2}$ and $\boldsymbol{h}_{1}$ as well as that between $\boldsymbol{h}_{2}$ and $h_{3}$. In other words, we cannot construct a variance estimation for $\beta(t)$ based on Theorem 3 directly. Of course, an alternative to this is to apply the bootstrap procedure and it is clear that a lot more work needs to be done.

\section{Supplementary Material}

The Supplementary Material includes extra simulation results, additional real data analysis results and the proof of the asymptotic theorems.

\section{Acknowledgments}

The authors wish to thank the Associate Editor and two anonymous referees for their many constructive and helpful comments and suggestions that greatly improved the 
paper. This research used the data from the China Health and Nutrition Survey (CHNS). The authors also want to thank the National Institute of Nutrition and Food Safety, China Center for Disease Control and Prevention, Carolina Population Center, the University of North Carolina at Chapel Hill, the NIH (R01-HD30880, DK056350, and R01-HD38700) and the Fogarty International Center, NIH for financial support for the CHNS data collection and analysis files from 1989 to 2006 and both parties plus the China-Japan Friendship Hospital, Ministry of Health for support for CHNS 2009 and future surveys.

\section{References}

Amorim, L. D., J. Cai, D. Zeng, and M. L. Barreto (2008). Regression splines in the time-dependent coefficient rates model for recurrent event data. Statistics in Medicine 27(28), 5890-5906.

Cook, R. and J. Lawless (2007). The Statistical Analysis of Recurrent Events. Springer-VerIag New York.

He, X., X. Feng, X. Tong, and X. Zhao (2017). Semiparametric partially linear varying coefficient models with panel count data. Lifetime Data Analysis 23(3), 439-466.

Hu, X. J., J. Sun, and L.-J. Wei (2003). Regression parameter estimation from panel counts. Scandinavian Journal of Statistics 30(1), 25-43.

Li, N., L. Sun, and J. Sun (2010). Semiparametric transformation models for panel count data with dependent observation process. Statistics in Biosciences 2(2), 191-210.

Lin, D. Y., L. J. Wei, and Z. Ying (2001). Semiparametric transformation models for point processes. 
Journal of the American Statistical Association 96(454), 620-628.

Lin, H., Z. Fei, and Y. Li (2015). A semiparametrically efficient estimator of the time-varying effects for survival data with time-dependent treatment. Scandinavian Journal of Statistics 43(3), 649-663.

Lu, M., Y. Zhang, and J. Huang (2009). Semiparametric estimation methods for panel count data using monotone B-splines. Journal of the American Statistical Association 104(487), 1060-1070.

Oliveira, J. (2016). The value of children: Inter-generational support, fertility, and human capital. Journal of Development Economics 120, 1-16.

Perperoglou, A. (2013). Cox models with dynamic ridge penalties on time-varying effects of the covariates. Statistics in Medicine 33(1), 170-180.

Sun, J. and X. Zhao (2013). Statistical Analysis of Panel Count Data. Springer New York.

Tian, L. (2018). Less is more: the effects of children on parents' income, assets and intergenerational transfers. Innovation in Aging 2, 468-468.

Tian, L., D. Zucker, and L. J. Wei (2005). On the cox model with time-varying regression coefficients. Journal of the American Statistical Association 100(469), 172-183.

Wang, Y. and Z. Yu (2021). A kernel regression model for panel count data with time-varying coefficients. Statistica Sinica 31, 1-19.

Wellner, J. A. and Y. Zhang (2007). Two likelihood-based semiparametric estimation methods for panel count data with covariates. The Annals of Statistics 35(5), 2106-2142.

Yu, Z. and X. Lin (2010). Semiparametric regression with time-dependent coefficients for failure time data 
analysis. Statistica Sinica 20(2), 853.

Zhao, H., W. Tu, and Z. Yu (2018). A nonparametric time-varying coefficient model for panel count data. Journal of Nonparametric Statistics 30(3), 640-661.

Department of Statistics, University of Missouri, 146 Middlebush Hall, Columbia, MO 65211 USA

E-mail: (yg882@mail.missouri.edu)

Department of Biostatistics and Bioinformatics, Emory University, 1518 Clifton Rd, Atlanta, GA 30322

USA

E-mail: (dayu.sun@emory.edu)

Department of Statistics, University of Missouri, 146 Middlebush Hall, Columbia, MO 65211 USA

E-mail: (sunj@missouri.edu) 